\title{
Single-destination navigation in a multiple-destination environment: a new "later-destination attractor" bias in route choice
}

\author{
En Fu ${ }^{1} \cdot$ Mary Bravo ${ }^{2} \cdot$ Beverly Roskos $^{1}$
}

Published online: 31 March 2015

(C) Psychonomic Society, Inc. 2015

\begin{abstract}
People choose different routes depending on the size of the environment. However, the size of the environment is often confounded by cognitive load. The current study investigated whether changes in route choice varied as a function of memory load while keeping the size of the environment constant. Also, a new situation was used: route choice to a single destination as a function of the location of follow-up destinations. Critically, there were two routes of equal length to the first destination. The results showed that route choice to the first destination was influenced by the locations of later destinations. This (new) "later-destination attractor" bias is the tendency to take a route to the first destination that begins in the direction of subsequent destinations even when the route to the first destination is logically independent of the locations of subsequent destinations. In Experiment 1, all destinations were visible, and the bias was seen for both second and third subsequent destinations. In Experiments 2 and 3, only the first destination was visible, and the locations of the subsequent destinations were indicated on a diagram and had to be remembered by participants. In Experiment 3, the diagrams were misaligned by 180 degrees. Route choice to the first destination was still influenced by later destinations, but only by the nearer, second destination, and the extent of the bias was reduced as memory load increased. Indications are that differences in route choice between small- and large-scale environments may be due more to cognitive load than to environmental size.
\end{abstract}

$\mathrm{En} \mathrm{Fu}$

efu@crimson.ua.edu

1 Department of Psychology, University of Alabama, Box 870348, Tuscaloosa, AL 35487, USA

2 Rutgers University, Armitage 345, Camden, NJ 08102, USA
Keywords Route choice $\cdot$ Route planning · Cognitive load · Later-destination attractor bias $\cdot$ Wayfinding

People engage in route planning many times every day, whether running errands on the way home from work or walking through a crowded hotel lobby. Because of the fundamental nature of this task, it has attracted the attention of cognitive psychologists who are interested in mental representations and in the link between perception and action (Allen, Kirasic, Rashotte, \& Haun, 2004; Downs \& Stea, 1973; Golledge, 1995; Hochmair \& Frank, 2000). The process of route planning has also attracted the attention of applied psychologists interested in aiding the design of comfortable, efficient, and safe environments (Gärling, Book, \& Lindberg, 1986; Raubal $\&$ Egenhofer, 1998). The research on route planning processes has typically focused on two complementary problems: how people plan the route between two locations and how they plan the sequence of the locations they wish to visit. The current studies, however, blend the two problems by focusing on the route people take between two locations when there are follow-up destinations in different locations. In addition, the current studies manipulate the memory load on the navigator, mimicking typical memory loads in different environments. Thus, two questions are addressed: Is the route taken to a first destination influenced by the locations of subsequent destinations? and does the choice of route change as a function of memory load? The goal is to explain why people choose the routes they take from one place to another.

\section{Cognitive biases at different environmental scales}

Previous research shows that both map-sized and life-sized environments are represented similarly in memory (Roskos- 
Ewoldsen, McNamara, Shelton, \& Carr, 1998). However, when the environment is large enough for navigation, and people can keep track of their location in the environment, representations of the space differ from that of a map-sized environment (Evans \& Pezdek, 1980; Presson, DeLange, \& Hazelrigg, 1989). Specifically, in life-sized spaces, people can imagine themselves in any place and in any orientation within the environment with the same ease, suggesting that the space is represented allocentrically. In contrast, with map-sized spaces, people find it difficult to imagine themselves in an orientation that differs from the orientation in which they learned the map (i.e., the map is represented egocentrically). Based on this, it is reasonable to expect that route choices may differ, depending on the size of the environment.

In an attempt to understand the effect of environmental size on navigation, Montello (1993) proposed four levels of psychological space: figural space (e.g., maps), vista space (e.g., rooms, yards), environmental space (e.g., cities, neighborhoods), and geographical space (e.g., cities within states, states within countries). Although Montello's categorization of space seems straightforward, the lines are blurred in actual studies. For example, Brunyé, Mahoney, Gardony, and Taylor (2010) investigated people's strategies for planning a trip in a real environment but used a Google map of the environment in their study. For our purposes, we discuss previous research as using small-scale spaces if participants could at least potentially see all locations simultaneously, and large-scale spaces if participants could not see all locations simultaneously.

Notice that the memory loads differ in these two types of environments. In small-scale environments, there is no need to remember locations because all locations can be seen. In large-scale environments, people must necessarily remember the locations of target destinations, assuming that no GPS is used. Most likely, people rehearse the location in short-term memory (STM), where STM is defined as a storage/rehearsal mechanism that lasts only a few seconds (e.g., Brown, 1958; Peterson \& Peterson, 1959; visual-spatial sketchpad in Baddeley, 1992). In some large-scale environments, you-arehere maps are the only (or easiest) way to locate target destinations, and people must translate the orientation of the map onto the actual locations in the environment. This translation, in addition to remembering the location of the target destination, presumably requires working memory (WM). Here, WM is defined as a "workbench" where STM interacts with longterm memory (LTM) that stores acquired knowledge and skills, and mental effort is involved (Baddeley, 1992; see also Shipstead, Lindsey, Marshall, \& Engle, 2014). Thus, most previous studies have confounded the size of the environment with the memory load involved when investigating route choices.

The current studies unconfounded environmental size and memory load by keeping the size of the environment static while manipulating the memory load of the task. Specifically, the study was conducted in a large room. In Experiment 1, all locations can be seen, thus representing a small-scale environment in which there is no memory load. In Experiment 2, only the first destination can be seen; the second and third were marked on a map. This set-up mimics a large-scale environment in which short-term memory is required to complete the task. Finally, Experiment 3 mimicked a you-are-here scenario: the locations of the second and third destinations are marked on a diagram and cannot be seen, and the diagram was misaligned relative to the actual locations in the room, thus requiring working memory to complete the task. The question is still, Which routes do people typically choose in small- and large-scale environments?

\section{Route choices in small-scale environments}

Most studies of route choice have used small-scale environments; typically, they are map-based, and so all destinations and possible paths are visible.

Single-destination studies in small-scale environments In studies where maps are used, participants typically choose among several possible routes from a starting location to a single target destination by drawing the route they would take. Participants tend to choose a path that has a long initial segment or the fewest turns (Bailenson, Shum, \& Uttal, 1998, Bailenson, Shum, \& Uttal, 2000; Christenfeld, 1995), and, most often, deviate the least from a straight line from start to destination (least angle; Hochmair \& Karlsson, 2005). In each case, participants seem to prefer the routes that require the least cognitive effort, even if the path is longer in the end (Wiener, Ehbauer, \& Mallot, 2009).

Other studies using single-destination small-scale spaces show participant preferences that are unique to the task. In one study, Brunyé et al. (2010) had participants study a map of a geographic space and then describe the most efficient path from one location to another location. When participants were planning a trip from east to west (or vice versa), they tended to choose a southern route. When planning a trip from north to south (or vice versa), their choices between an eastern or western route were at chance. Follow-up studies indicated that the southern bias was due to a misperceived elevation of the northern part of the map. This route selection was termed a north-up bias. Another study was conducted in the book stacks of an actual library (Scharine \& McBeath, 2002). Participants from different countries stood at one end of an aisle between book stacks and were told that there was a target at the other end of the aisle on either the left or the right side. U.S. participants consistently preferred to turn right upon reaching the end of the aisle, presumably because most people are right-handed and because driving is on right side of the road in the U.S. This finding represents a right-turn bias. 
Multiple-destination studies in small-scale environments Multiple-destination studies often use the traveling salesman problem (TSP), where a salesman (the participant) has to stop at a number of locations. On a map that shows all locations, or in a virtual environment in which all locations can be seen, participants show the path they would take. In some cases, participants choose a convex arch of all of the outer locations first, then go to the inner locations (convex hull bias; MacGregor, Chronicle, \& Ormerod, 2004; MacGregor \& Ormerod, 1996). In other cases, they visit the closest location first, then the next closest location, and so on (nearest neighbor bias; Vickers, Bovet, Lee, \& Hughes, 2003). In still other cases, participants choose to visit the nearest cluster of locations first, then the next nearest cluster, and so forth (hierarchical clustering; Graham, Joshi, \& Pizlo, 2000). Finally, when there are marked regions on a map or in a virtual neighborhood and the starting and ending locations are in different regions, participants prefer the path that gets them to the edge of the first region the quickest, even if other routes are shorter in distance to the end location (Wiener et al., 2009). This regionalization bias especially reflects a conservation of cognitive effort: just get to the next region quickly, and then worry about the location of the destination(s) within the second region. Further, Wiener et al. found that as memory demand increased, participants were less likely to take the optimal/shortest route.

\section{Route choices in large-scale environments}

In all of the small-scale situations, participants could see the destination and all possible routes to it, and there was no need to remember the target location. In these situations there were multiple biases in play depending on the complexity of the environment. In environments where the destination is not directly visible from the starting location, route choices may differ from those in smallscale environments.

\section{Single-destination studies in large-scale} environments Researchers using large-scale spaces often use virtual environments; for example, participants might choose a route to a destination within a virtual city (Hochmair, 2005; Hochmair \& Karlsson, 2005) or navigate a virtual environment of urban block shapes with the goal of walking "to the opposite corner by the most direct route possible" (Dalton, 2003, p. 107-131). Results from these studies showed that participants tried to conserve the linearity of their routes (i.e., they showed a least angle bias). Other researchers using large-scale spaces have found that participants have a regionalization bias. In one study that involved clearly identified neighborhoods, participants chose the quickest path to the edge of their neighborhood when navigating to a location in another neighborhood (Hochmair, Büchner, \& Hölscher, 2008; see also Timmermans, van der Hagen, \& Borgers, 1992; Wiener \& Mallot, 2003). The same bias is seen when choosing paths to a destination within a multiple-story building (Hölscher, Meilinger, Vrachliotis, Brösamle, \& Knauff, 2006). A variation on the regionalization bias is seen in a study of experienced taxi drivers (Spiers \& Maguire, 2008). The taxi drivers in this study tended to carve their cities into regions and would plan a route to the edge of one region, then pause and plan a route to the edge of the second region, and so on, This hierarchical regionalization bias allowed them to conserve cognitive effort by only keeping one intermediate destination in mind, instead of remembering the entire route (see also fine-to-coarse bias; Wiener \& Mallot, 2003).

Multiple-destination studies in large-scale environments Wiener, Schnee, and Mallot (2004) is the classic study investigating route choices in simulated large-scale environments. Their virtual world comprised islands with landmarks that popped up when approached. The regions were carefully constructed to tease apart a fine-to-coarse bias (i.e., hierarchical regionalization), in which they find their way through one region before thinking about a route through other regions, and a least-decision-load bias, in which the route is chosen to minimize complexity. Across seven different types of target arrangements, the least-decision-load bias predicted participants' routes in 6 of 7 , whereas the fine-tocoarse bias predicted participants' routes in 5 of 7 . These results indicate that these two biases have considerable overlap and that conserving memory load is important in these situations.

\section{Summary and hypotheses}

In the simplest of navigation tasks - a single visible destination-many different biases are seen in participants' route choices. Most of these biases appear to conserve physical effort (travel time or distance). These heuristics include choosing the route with the longest initial segment or choose the route that deviates the least from the direct path (i.e., the route with the least angle). As the navigation task becomes more complex, either because the destination is not visible or because there are multiple destinations, the predominant route choices are those that conserve cognitive effort. In particular, navigators tend to regionalize the environment and make decisions first about how to get out of the first region and then how to get through the next region, and so on. In the current studies, this suggests that participants may use a least angle strategy that focuses on nearer destinations as memory load increases. 


\section{Experiment 1}

To examine the effects of later locations on route choice to the first destination, we created a task that required visiting, in predetermined order, three destinations in a room. Participants sat at a reception table facing the interior of a room. In Experiment 1, participants were able to see all of the destinations and were told the order in which they should be reached. To get to the first destination, which had the same location on all trials, they had to go around the reception table, creating the main dependent measure: whether they went around the left side or the ride side of the reception table. Both routes were the same length and had the same angle away from the destination. The route segments were relatively independent of each other, and so there was no reason for the location of the intermediate or final destinations to affect the route to the first destination.

If the locations of later destinations had no impact on the route choice to the first destination, one would expect the choice of routes (right or left) to be at chance (50\%). If anything, one may see a right-turn bias (e.g., Scharine $\&$ McBeath, 2002). If the locations of later destinations influenced the route choice to the first destination, there are two possibilities: Participants might align their initial path in the direction of the second destination or they might align their initial path in the direction of the final destination.

\section{Method}

Participants A power analysis using $\mathrm{G} *$ Power 3.1.9.2 (Faul, Erdfelder, Lang, \& Buchner, 2007) was conducted to determine the number of participants needed, using the ANOVA: repeated measures, within factors protocol, $\alpha=.05, \beta=.80$, effect size $f=.36,{ }^{1} \#$ groups $=1$, $\#$ measures $=3$ (left, middle, right), which suggested $N=14$. The sample size was rounded up to 20 to afford a linear trend analysis using the rule of thumb of 10 participants per predictor variable (second, third destinations).

Twenty-two participants (average age $=21 ; 13$ women and 1 unidentified) were recruited from students in the Introduction to Psychology class at Rutgers UniversityCamden campus, either for a $\$ 10$ payment or to fulfill a class requirement.

\footnotetext{
${ }^{1}$ The effect size was based on an average of effect sizes from Brunyé et al. (2010; Experiments 1 and 2). The effect sizes came from tests in which a difference was expected. In Experiment 1, effect sizes were $d=$ $.81, .45,1.11$, and .79. In Experiment 2, they were $d=.46, .83$, and .60 . The average effect size $(d=.72)$ was converted to $f=.36$ for use in a three-condition one-way ANOVA using the conversion calculator found at http://www.stat-help.com.
}

Experimental set-up The experiment took place in a basement conference room with no windows. The room contained three rows of tables along with a reception table (see Fig. 1A). The room was $10.21 \mathrm{~m}$ wide $\times 7.32 \mathrm{~m}$ deep. The tables were $1.68 \mathrm{~m}$ wide $\times 0.61 \mathrm{~m}$ deep $\times 0.74 \mathrm{~m}$ tall. The distances between the rows were $1.07 \mathrm{~m}$ for the first and second rows, $1.22 \mathrm{~m}$ for the second and third rows, and $0.30 \mathrm{~m}$ for the third row and wall. The spacing between the tables within rows 1 and 2 was $.91 \mathrm{~m}$, with $1.68 \mathrm{~m}$ between the outside tables and the wall. For the third (last) row, the tables formed a continuous row, with $.91 \mathrm{~m}$ between the outside tables and the walls.

On some of the trials, only two rows were used, creating a two-destination route. On the other trials, all three rows were used, creating a three-destination route. On one table in each of the first two (or all three) rows was a colored piece of paper that indicated a destination (see Fig. 1B). Each destination was indicated by a different paper color, and the colors were randomized across trials. On each paper there was a single multiple-choice question related to risk-taking or organizational behavior (e.g., "When I have lost something valuable and can't find it anywhere: A. I have a hard time concentrating on anything else, or B. I don't dwell on it."). Most questions were repeated several times during a session. The main purpose of the surveys was to cause the participants to stop for a moment at each destination, but the surveys also provided a "cover" for the experiment.

The location of the first destination was fixed - it was always on the center table of the first row (labeled X in Fig. 1B). The second destination was located in the second row on either the left, middle, or right table. On three-destination trials, the third destination was on the left, middle, or right table of the third row. In all cases, the participant could see all of the colored papers, and their only task was to go from one piece of paper to the next. Participants were told that the aim of the experiment was to explore human decision-making behavior in real environments. They were given no indication that their route choices were being measured.

The experimental design of the two-destination trials consisted of three combinations: the second destination could be on the left, middle, or right table. In the three-destination there were 3 (second-row destinations) $\times 3$ (third-row destinations) $=9$ combinations. Each participant completed two blocks of trials; in each block, all 12 combinations were tested, for a total of 24 trials. We divided the experimental session into two blocks for convenience. The orders of the trials within the first block and the second block were randomly determined (i.e., random selection from a full permutation list). The direction that the participant went around the reception table (right or left) to get to the first destination was recorded for each trial.

Procedure Each participant was tested in a single 50-minute session. The participant began the session at the reception 
A

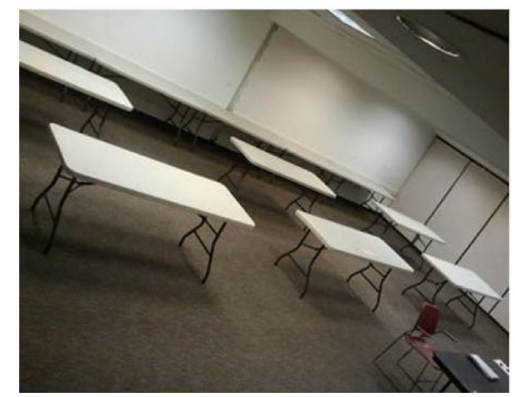

B

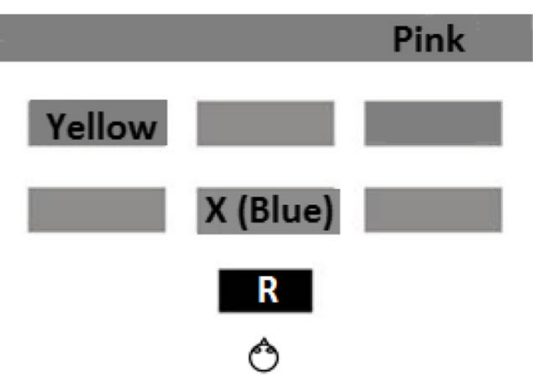

Fig. 1 Experiment 1: The physical layout of the room (A), and one combination of a three-destination route: Blue, Yellow, and Pink (B). The reception table is labeled R. The first destination is always located in the center of the first row of tables, here labeled X

table in the front of the room. The participant was seated facing the experimenter and the rows of tables. After completing the consent form, the participant was given the following instructions: "Remain seated and take a look at the papers behind me on the tables. You are going to reach them in order from the first row to the last row. Each time you reach a paper, answer the question on it. Before you leave this reception table, please tell me the colors of the papers in order." We elicited this response so that the participants would attend to the destination order.

Then the participants walked to each destination in turn and answered the multiple-choice question at the destination. In particular, they walked to the center of the table on which the paper was located. The multiple-choice question at each destination was randomly picked from Scale One, which had eight questions, or from Scale Two, which had 16 questions. After answering the question at the final destination, the participant returned to the seat at the reception table. To discourage participants from watching the experimenter set up the next trial, they were asked to copy a detailed drawing. Participants completed all possible combinations of second and third destinations in a single block (Block 1; 3 twodestination trials +9 three-destination trials), then completed each combination a second time (Block 2; another 12 trials). After both blocks were completed, participants were asked what they thought the experiment was about. Most participants who tried to guess the purpose thought it examined the effect of the paper color on their answers to the survey questions; none of the participants thought the study examined route choice.

\section{Results and discussion}

Before analyzing the data, we first checked for error trials. If a person did not take the shortest route to the second destination (e.g., they were to walk to the middle table but they walked to the left table and then to the middle table), the trial was considered to be an error trial. There were 264 trials in each block (12 trials $\times 22$ participants). In Block 1 the number of error trials was $0(0 \%)$; in Block 2 there were 2 error trials $(.8 \%)$. Overall, percentage of errors in the current experiment was extremely low, which would be expected because participants can see all of the destinations. The pattern of results were the same with or without these error trials; they were included in the analyses to preserve power (i.e., the results were actually stronger when they were included).

For the main analyses, a left route around the reception table to the first destination was coded as " 0 " and a right route was coded as " 1 ." Then, the two responses for each possible combination were averaged and multiplied by 100 , producing the percentage of times the participant chose the right route around the reception table. Averaging across participants, a mean closer to 0 means that they had a bias to go left (i.e., a left bias), and a mean closer to 100 means they had a bias to go right (i.e., a right bias); an average near 50 means that path choice was at chance.

The data from the two-destination trials - those without a third destination-were submitted to a general linear model analysis with one repeated measures factor (second destination: left, middle, right). Figure $2 \mathrm{~A}$ shows that the percentage of times that participants took the right route around the reception table increased steadily across the left, middle, and right destinations, main effect: $F(2,40)=18.50, M S E=$ $.071, p<.001$, part $\eta^{2}=.48$; linear trend: $F(1,20)=35.00$, $M S E=.075, p<.001$, part $\eta^{2}=.64$. When the second destination was on the right, participants were over three times more likely to select the right route $(71 \%)$ than when the second destination was on the left $(21 \%)$. When the second destination was in the middle, the percentage of routes taken to the right around the reception table was at chance. Clearly, the route that participants took to the first destination was influenced by the location of a subsequent second destination, indicating a least-angle bias.

This effect persisted over multiple segments. The data from the three-destination trials were submitted to a general linear model analysis with a 3 (second destination) $\times 3$ (third destination) factorial model and repeated measures on both factors. There was a main effect of the second destination as well as a 
Two-Destination Trials

A
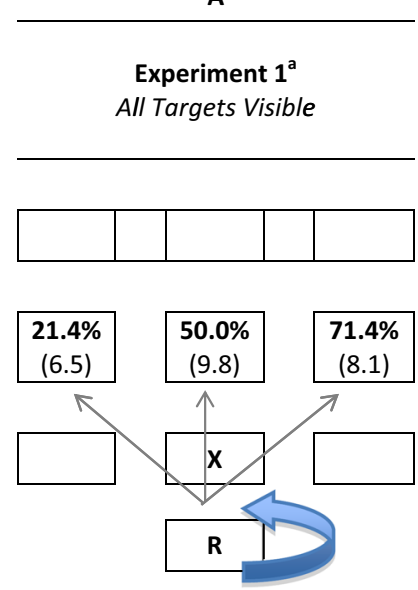

Fig. 2 Results as a function of second destination on two-destination trials for Experiments 1 (A), 2 (B), and 3 (C). Estimated marginal means (and standard errors) of the percentage of trials on which participants chose the right path around the reception table $(\mathrm{R})$ as a function

linear trend, just as there was when there was only two destinations, main effect, $F(2,40)=21.41, M S E=.118, p<.001$, part $\eta^{2}=.52$; linear trend, $F(1,20)=30.63, M S E=.156, p<$ .001 , part $\eta^{2}=.61$ (see Fig. 3A). When the destination was on the right, participants were almost three times more likely to take the right route around the reception table (59\%) than the left route $(20 \%)$. When the second destination was in the middle, participants took the route to the around half the time. There was a main effect of the third destination, and a linear trend, main effect, $F(2,40)=5.27, M S E=.081, p=.009$, part $\eta^{2}=.21$; linear trend, $F(1,20)=6.10, M S E=.105, p=.023$,
$B$

Experiment $2^{\mathrm{b}}$ $2^{\text {nd }} \& 3^{\text {rd }}$ Targets Hidden ocations on Diagram

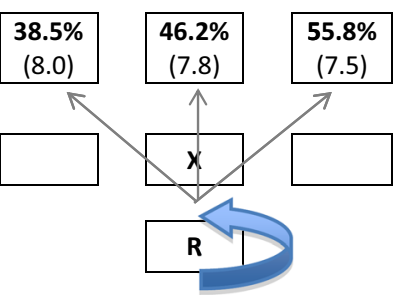

C
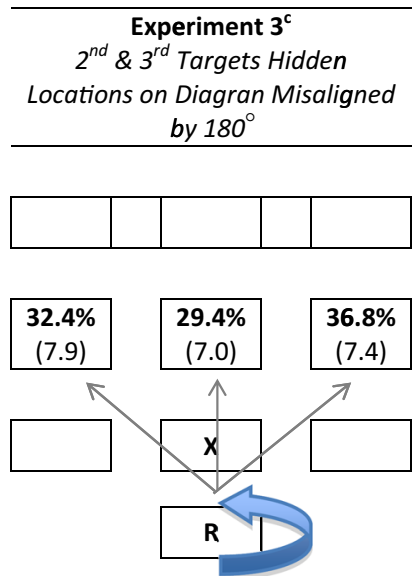

of the second destination. The first destination was always in the middle of the first row of tables (X). Chance is $50 \%$. ${ }^{\mathrm{a}}$ Main effect of second destination; significant linear trend. ${ }^{\mathrm{b}}$ No main effect; significant linear effect. ${ }^{\mathrm{c}}$ No main effect; no linear effect

part $\eta^{2}=.23$ (see Fig. 4A). When the third destination was on the left, they were more likely to take the left route than the right route around the reception table; otherwise their choice was near chance.

Finally, there was an interaction of the second and third destinations on route preference that undermines the main effect of the third destination, $F(4,80)=2.90, M S E=.042$, $p=.027$, part $\eta^{2}=.13$. To understand the interaction, three one-way repeated measures ANOVA, with the locations of the third destination as levels of the IV, was calculated for each second destination. To reduce experiment-wise error when

Three-Destination Trials - Second Destination Only

A

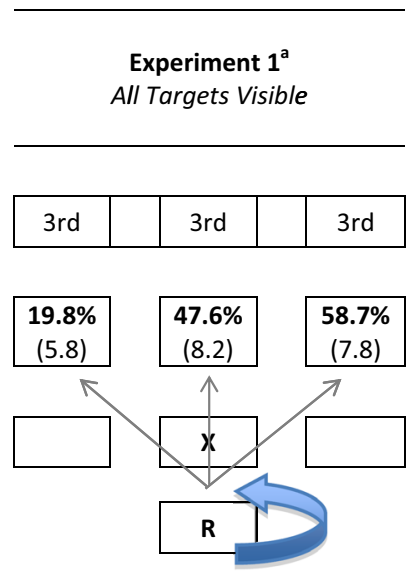

B

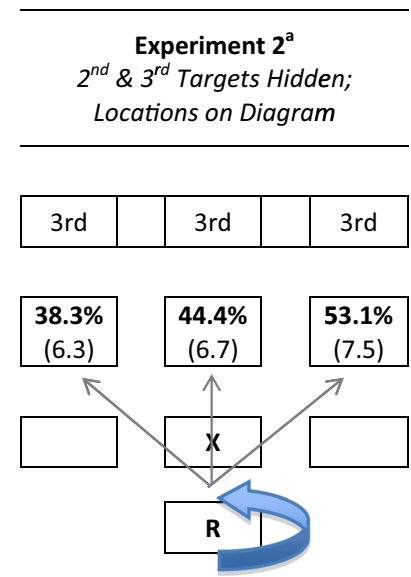

C

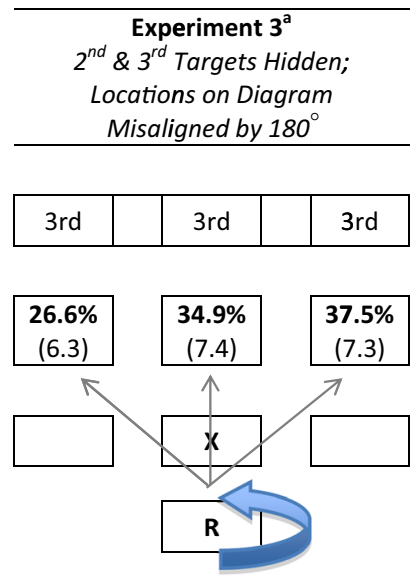

Fig. 3 Results as a function of second destination, regardless of third direction, on three-destination trials for Experiments 1 (A), 2 (B), and 3 (C). Estimated marginal means (and standard errors) of the percentage of trials on which participants chose the right path around the reception table
(R) as a function of the second destination. The first destination was always in the middle of the first row of tables (X). Chance is $50 \%$. ${ }^{\text {a Both }}$ main and linear effects are significant 
Three-Destination Trials - Third Destination Only

A

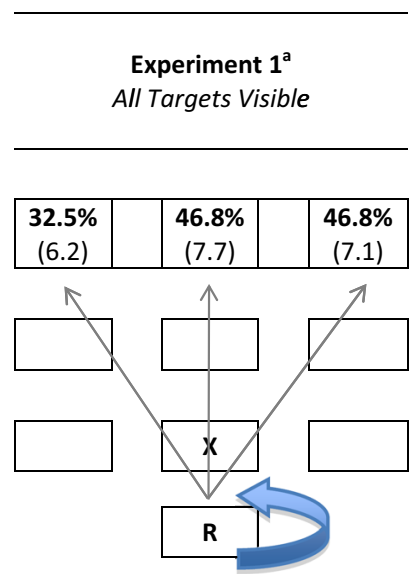

Experiment $\mathbf{1}^{\mathrm{a}}$

All Targets Visible

Fig. 4 Main effect of third destination, regardless of second destination, on three-destination trials for Experiments 1 (A), 2 (B), and 3 (C). Estimated marginal means (and standard errors) of the percentage of trials on which participants chose the right path around the reception table (R), as a function

completing multiple analyses on the same data, the acceptable probability of making a Type I error was reduced by one-third (i.e., $p<.0167)$. When the second destination was to the left, the percentages did not differ, $F(2,42)=2.92, M S E=.056, p$ $=.065$, part $\eta^{2}=.12$, and the increase from left to right was not linear, $F(1,21)=.14, M S E=.041, p=.72, . \operatorname{part} \eta^{2}=.01($ see Fig. 5A). No matter where the third location was, participants chose the left route more often than the right to get around the reception table to the (middle) first and (left) second destinations.

When the second destination was in the middle, there was a main effect of the third destination on route preference around the reception table, and a linear trend, main effect, $F(2,40)=$ 8.68, MSE $=.049, p=.001$, part $\eta^{2}=.30$; linear trend, $F(1,20)$ $=15.00, M S E=.057, p=.001$, part $\eta^{2}=.43$ (see Fig. 5B).
B

xperiment $2^{\mathrm{a}}$

\& $3^{\text {rd }}$ Targets Hidden

cocations on Diagram

\begin{tabular}{|c|c|c|}
\hline $\begin{array}{c}\mathbf{4 5 . 7 \%} \\
(5.9)\end{array}$ & $\begin{array}{c}\mathbf{4 5 . 1 \%} \\
(6.3)\end{array}$ \\
\hline
\end{tabular}

$\uparrow \quad 7$

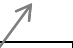

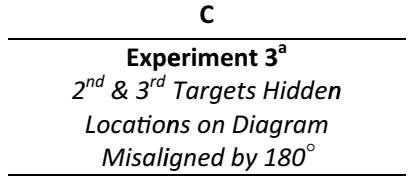

\begin{tabular}{|c|c|c|c|c|}
\hline $\begin{array}{c}\text { 30.2\% } \\
(6.7)\end{array}$ & $\begin{array}{c}\mathbf{3 3 . 3} \% \\
(6.8)\end{array}$ & $\begin{array}{c}\mathbf{3 5 . 4 \%} \\
(7.2)\end{array}$ \\
\hline
\end{tabular}

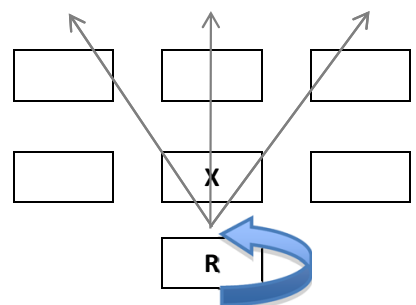

of the third destination. The first destination was always in the middle of the first row of tables (X). Chance is $50 \%$. ${ }^{\mathrm{a}}$ Both main and linear effects are significant (but see Fig. 5 for interaction). ${ }^{\text {b }}$ Neither main effect nor linear effect are significant
Similar to the two-destination results, when the third destination was on the right, participants chose the right route almost twice as often $(62 \%)$ as when the third destination was on the left $(33 \%)$. When the third destination was in the middle, route preference was around chance level. Last, when the second destination was on the right, participants were more likely to select the right route around the reception table no matter where the third destination was located; there was neither a main effect for the third destination nor a linear trend, main effect, $F(2,42)=.64$, MSE $=.077, p=.53$, part $\eta^{2}=.03$; linear trend, $F(1,21)=.42, M S E=.123, p=.53$, part $\eta^{2}=.02$ (see Fig. 5C).

Overall, it is clear that the location of the second destination influenced the route that participants took around the reception table to get to the first destination, which was always in

\section{Three Destination Trials - Experiment 1 Interaction}
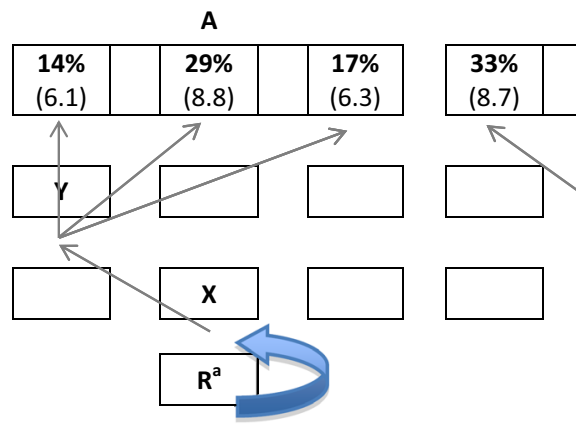

Fig. 5 Experiment 1: Interaction of second and third destinations on three-destination trials. Estimated marginal means (and standard errors) of the percentage of trials on which participants chose the right path around the reception table (R), when the second destination was on the left (A), in the middle (B), and on the right (C). The first destination was
B

\begin{tabular}{|l|l|l|}
\hline $48 \%$ & $62 \%$ \\
\hline
\end{tabular}

(9.4) $\quad(9.1)$
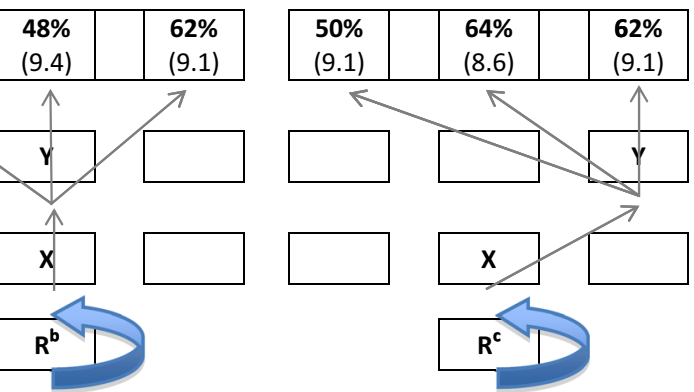

always in the middle of the first row of tables (X). The second destination

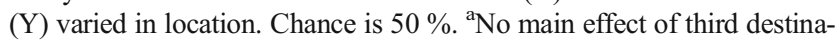
tion; no linear effect (significant quadratic). ${ }^{b}$ Main effect of third destination; linear effect. ${ }^{\circ}$ No main effect of third destination; no linear effect 
the middle. The third destination had some influence but it depended on the location of the second destination, with each second destination producing a different pattern of route preferences. When the second destination was on the left, there was a left-route bias for all three third-destination locations, and when the second destination was on the right there was a right-route bias for all three third-destination locations. When the second destination was in the middle there was a split in route preference: there was a preference to go left when the third destination was on the left and a preference to go right when the third destination was on the right.

In this experiment set-up, the first destination was always placed at the center of the middle table in the first row. The length of the left route and right route were equal, and thus the probability for participants to choose the left and right routes to the first destination should be equal if the route segments were planned independently. The results from this experiment, however, clearly show that route segments are not planned independently. Rather, the route navigators chooses to take to a particular destination is not determined solely by the characteristics of the routes to that destination; it is also determined by the location of subsequent destinations, at least when they can see all destinations. In particular, it appears that participants had a least-angle bias for the location of the second destination. The third destination had no impact on their path choice to the first destination in these cases. The location of the third destination only influenced participants' path choice when the second destination was in the middle, a case in which they would normally choose the left and right route around the reception table equally. Here, it makes sense that a later destination would be the focus of a least-angle bias.

The next experiment examines whether participants' route preferences hold when they need to use STM to remember the locations of later destinations.

\section{Experiment 2}

The results from Experiment 1 are consistent with the idea that when navigators set out on a route with multiple segments, their choice for the first route segment is biased not only by the direction of the first destination, as others have demonstrated, but also by the direction of subsequent destinations. Of course, these directions are especially clear and salient when the goal destinations are visible, and the success of Experiment 1 may have hinged on this visibility. The powerful draw of visible goals has been demonstrated by studies involving animals and children, who have difficulty moving away from a goal they can see, even when moving away from this goal is the fastest (or only) way to reach the goal (Lockman, 1984; Vlamings, Hare, \& Call, 2010).

The objective of Experiment 2 was to introduce a STM load to the task. We repeated the previous experiment while blocking the participants' view of everything after the first destination. Participants were provided with a diagram showing where each destination would be on that specific trial. Because the location of the third destination was visible once they reached the second destination, it was not necessary for them to remember its location. In this case, we might expect a least-angle bias to the second destination and a diminished or absent effect of location of the third destination, reflecting a tendency to conserve cognitive effort.

\section{Method}

Participants The same power analysis as in Experiment 1 was used for Experiment 2. To increase the power of detecting a linear trend for the third destination, the number of participants was increased to a goal of 30 . Twenty-eight participants (average age $=19 ; 15$ women and 1 unidentified) were recruited from students in the Introduction to Psychology class at Rutgers University-Camden campus either for a $\$ 10$ payment or to fulfill a class requirement.

Experimental set-up and procedures The experimental setup and procedures were similar to those of the first experiment, with two exceptions. First, room dividers were positioned between the first and second rows of tables (see Fig. 6A). Gaps between the dividers allowed participants to cross the rows, but the gaps were not sufficient to allow participants to see the papers located in the second and third rows. Second, participants learned the locations of these destinations from a diagram that they viewed while seated at the reception table (see Fig. 6B). The color of the paper at each destination was also indicated on the diagram. To ensure that the participants paid attention to each destination on the diagrams, they were asked to say aloud the colors of the papers in the order that they would visit them.

\section{Results and discussion}

Before analyzing the data, we first checked the number of errors trials. There were 336 trials in each block (12 trials $\times$ 28 participants). In Block 1 there were $0(0 \%)$ error trials and in Block 2 there were $3(.9 \%)$. As in Experiment 1, errors were extremely low. The pattern of results was the same with or without these error trials, so they were included in the analyses to preserve power.

The data from the two-destination trials - those without a third destination - were submitted to a general linear model analysis with one repeated measures factor (second destination: left, middle, right). On the two-destination trials, the results (see Fig. 2B) were similar to those in Experiment 1, with a couple of exceptions. First, the differences in path choice when the second destination was on the right versus the left were much smaller in Experiment 2 than in 
A

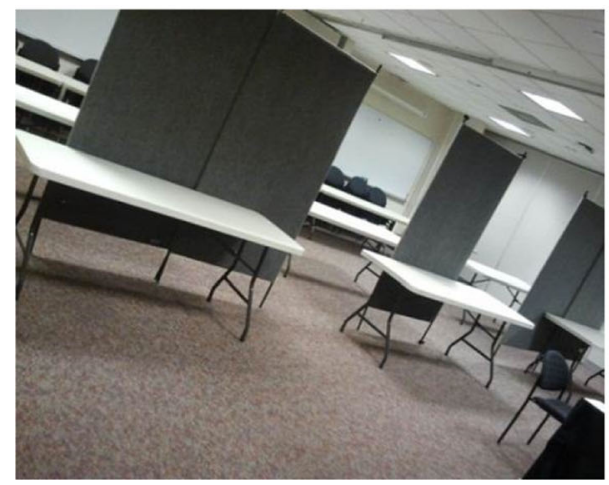

B

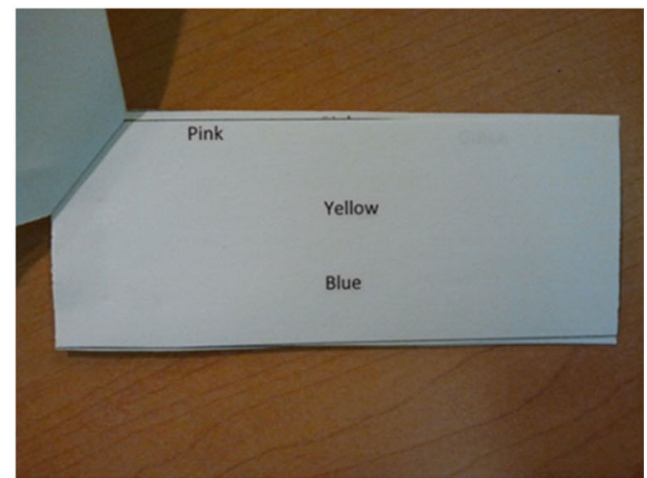

Fig. 6 Experiment 2: The physical layout of the room with dividers between the first and second destinations (A), and the diagram showing the locations of the destinations (B)

Experiment 1; in fact, there were no significant differences in Experiment $2, F(2,50)=2.59, M S E=.076, p=.085$, part $\eta^{2}=$ .09 . However, the linear trend was significant, $F(1,25)=4.90$, $M S E=.079, p=.036$, part $\eta^{2}=.16:$ the percentage of times participants chose the path around the right side of the reception table steadily increased depending on the location of the second destination (left, middle, right). Second, participants in Experiment 2 seemed to choose the left path when the second destination was in the middle ( $46 \%$ right path) more often than in Experiment 1 (50\% right path). Only when the second destination was on the right did participants choose the path to the right (56\%).

To test whether the route choices were significantly different from chance ( $50 \%$ ), we calculated three averages: one for each location of the second destination, regardless of the location of the third destination (if there was a third destination). When the second destination was on the left, the percentage of times participants chose the right path around the reception table was $38 \%$, which approaches a significant difference from chance, $t(27)=-1.97, p=.06$, two-tailed. Neither the middle second destination nor the right second destination differed significantly from chance. However, there was a significant difference between route choices for the left seconddestination trials and right second-destination trials, $t(27)=-$ $2.39, p=.024$. The other pairs were not statistically different, left vs. middle, $t(27)=-1.65, p=.11$; middle vs. right, $t(27)=$ $1.61, p=.12$.

The data from the three-destination trials were submitted to a general linear model analysis with a 3 (second destination) $\times 3$ (third destination) factorial model and repeated measures on both factors. For the threedestination trials, there was a linear trend for the second destination, $F(1,26)=4.52, M S E=.197, p=.043$, part $\eta^{2}$ $=.15$, with an increasing preference for the path around the right from left to middle to right (see Fig. 3B). However, compared to Experiment 1, overall differences between the locations were reduced, $F(2,52)=3.18, M S E$
$=.141, p=.05$, part $\eta^{2}=.11$. And, participants had a bias to take the left route around the reception table, except when the second destination was on the right (53\%).

In contrast with the previous experiment, however, the location of the third destination did not have a direct influence on route choice towards the first destination, either as a main effect or a linear trend, main effect, $F(2$, $52)=.02, M S E=.059, p=.98$, part $\eta^{2}=.001$; nor did it interact with the second destination, $F(4,104)=1.51$, $M S E=.084, p=.20$, part $\eta^{2}=.06$. Figure 4B shows that there was a left-side bias regardless of the location of the third destination.

Overall, the effects of subsequent destinations on route planning towards the immediate destination were similar but less pronounced than those in Experiment 1. Clearly, participants focused more on the second destination than the third, which fits best with a regionalization bias, but there was an overall left bias except when the second destination was on the right. This result is in the opposite direction as that expected from the right-bias literature. Although the subsequent destinations were hidden from direct view in Experiment 2, their directions were still easy to ascertain from the diagram and could be easily rehearsed in STM. Nonetheless, participants' performance suggested that although they remembered the second destination to some extent, they did not seem to be remembering the third destination to any extent.

This second experiment examined whether the route choice to an immediate (first) destination was affected by the location of subsequent destinations when the participant had to remember the locations of the later destinations. The results indicated that hidden distant destinations still had an effect on route choice to immediate destinations. The final question was whether this effect would be found when the memory load was even higher. In the final experiment, we increased the memory load even further by requiring WM. 


\section{Experiment 3}

The previous experiments demonstrated that when participants navigated to multiple predetermined destinations, their choice for the initial route segment was biased by the direction of distant destinations. The first experiment showed that this effect is strong when the distant destinations are visible. The second experiment showed that this effect still occurs for the second destinations when the destinations are not visible but their directions are readily obtained from a diagram (i.e., using STM). In this third experiment, we examined whether this bias towards the direction of a distant goals persists even when this direction must be derived from an upside-down diagram, using $\mathrm{WM}$, and the distant locations were not visible. The upside-down diagram had the same information content as the diagram in the previous experiment, but this information was no longer directly compatible with the participant's egocentric reference frame. Instead, participants had to align the diagram so that the top of the diagram faced the far side of the room and then map the locations on the diagrams to the locations in the room. In this case, participants may have a least-angle bias to the second region (the second destination). It is doubtful that they would have a least-angle bias for the final (third) destination because they did not do so when they were required to simply remember the locations for a short period of time in Experiment 2. Finally, there is the question of the left-side bias seen in Experiment 2. Perhaps this finding was an anomaly; if so, we probably would not see it in Experiment 3.

\section{Method}

Participants The same power analyses as in Experiments 1 and 2 was used for Experiment 3. Thirty-five participants (average age $=21 ; 17$ women) were recruited from students in the Introduction to Psychology subject pool at Rutgers University-Camden campus either for a $\$ 10$ payment or to fulfill a class requirement.

Experimental set-up and procedures The general layout and procedures were the same as those in the previous experiments, with the following exceptions. Shoe boxes were placed on the tables with the open side up at each of the six possible locations for the second and third destinations, and the surveys were placed inside the boxes (see Fig. 7A). Although the boxes were open at the top, a participant would need to peek inside the box to determine if it contained a survey. Thus, the boxes not only hide the destinations from the participants but also allow the experimenter to determine whether a participant knew where the surveys were located. Before starting on their multiple-destination trips, the participants were informed of the survey locations using the same diagram as in Experiment 2, but now these diagrams were presented with an upside-down orientation (see Fig. 7B). Thus, the destinations' directions on the diagrams were misaligned by 180 degrees with the participant's view of the room. To complete this task efficiently, participants needed to correctly encode the locations of the destinations indicated in the diagrams as well as align the "top" of the diagram with the far wall.

The conference room that was used for the first two experiments was unavailable for this third experiment, and so this experiment was conducted in a very similar room using the same tables in the same arrangement as those in the previous experiments.

\section{Results and discussion}

First, the number of error trials was calculated. Unlike the first two experiments, participants in this experiment had some difficulty remembering the location of the second destination. As a result, we analyzed separately the two-destination and three-destination trials as well as Block 1 and Block 2. In each block there were 105 two-destination trials ( 3 trials $\times 35$ participants), and 315 three-destination trials ( 9 trials $\times 35$ participants). On the two-destination trials, participants did not walk directly to the second destination from the first destination on two error trials in Block 1 (1.9\%) and two trials in Block 2 $(1.9 \%)$. On the three-destination trials, there were 17 error trials in Block $1(5.4 \%)$ and seven error trials in Block 2 $(2.2 \%)$. Nonetheless, the overall percentage of errors in the current experiment was low. The pattern of results was the same with or without these error trials, so they were included in the analyses to preserve power.

The data from the two-destination trials were submitted to a general linear model analysis with one repeated measures factor (second destination: left, middle, right). For the twodestination trials, there was neither a main effect of the location of the second destination on route preference around the reception table nor a linear trend (see Fig. 2C), main effect, $F(2,66)=.95, M S E=.049, p=.39, \operatorname{part} \eta^{2}=.03 ;$ linear trend, $F(1,33)=.59, M S E=.056, p=.45$, part $\eta^{2}=.02$. Furthermore, there was a marked left-side bias in all conditions.

To test whether the route choices were significantly different from chance $(50 \%)$, we calculated three averages: one for each location of the second destination, regardless of the location of the third destination (if there was a third destination). When the second destination was on the left, the percentage of times participants chose the right path around the reception table was $26 \%$, which is significantly different than chance, $t(34)=-4.12, p<.001$, two-tailed. When the second destination was in the middle, the percentage was $35 \%$, which differs from chance, $t(34)=$ - 
A

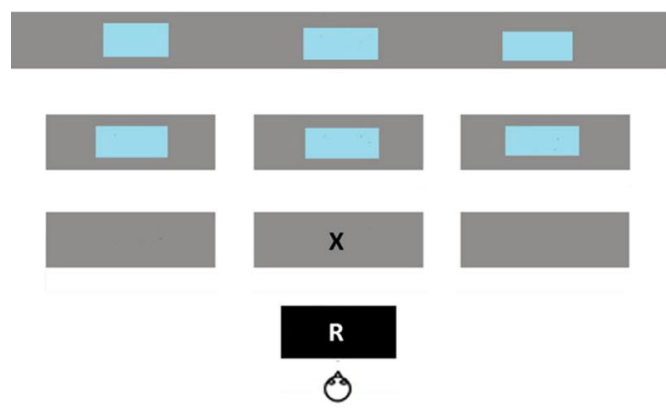

B

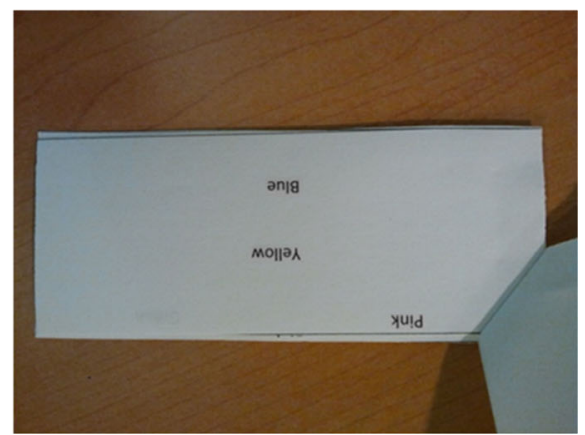

Fig. 7 Experiment 3: The physical layout of the room with shoe boxes hiding the second and third destinations (A), and the inverted diagram showing the locations of the destinations $(\mathrm{B}) . \mathrm{R}=$ reception table; $\mathrm{X}=$ first destination

$2.23, p=.032$, two-tailed. Only when the second destination was on the right route ( $39 \%$ ) was the difference not significant compared to chance, $t(34)=-1.64, p=.11$.

The data from the three-destination trials were submitted to a general linear model analysis with a 3 (second destination) $\times$ 3 (third destination) factorial model and repeated measures on both factors. In contrast to the two-destination trials but similar to the findings of Experiments 1 and 2, there was a main effect for the second destination and a linear trend (see Fig. 3C), main effect, $F(2,62)=4.08, M S E=.077, p=$ .022 , part $\eta^{2}=.12$; linear trend, $F(1,31)=5.26, M S E=$ $.109, p=.029$, part $\eta^{2}=.15$. The percentage of times the route preference was to the right steadily increased from the left, to the middle, to the right destinations. However, all of the percentages fell below $40 \%$, meaning that there was a strong left bias overall. For the third destinations, there was neither a main effect on route preferences around the reception table nor a linear trend (see Fig. 4C), main effect, $F(2,62)=1.26, M S E=.053, p=.29$, part $\eta^{2}=.04$; linear trend, $F(1,31)=1.83, M S E=.071, p=.19$, part $\eta^{2}=$ .06 . No matter where the third destination was, participants tended to begin their route around the left side of the reception table (all percentages $<40 \%$ ). There was no interaction between the second and third destinations, either, $F(4,124)=1.11, M S E=.039, p=.36$, part $\eta^{2}=.03$.

Similar to the results of Experiment 2, participants seemed to focus on the second region for route planning - in this case, the second destination. Also similar to Experiment 2, participants tended to choose the left route around the reception table overall, in contrast to the right-side bias found in earlier studies (Scharine \& McBeath, 2002). The right-side bias had been attributed to handedness; compared to left-handers, righthanders are more likely to turn right. Although handedness was not gathered for our participants, around $90 \%$ of Americans are right-handed, so it is likely that most of the participants in the current studies were right-handed. It is unclear why this overall left bias occurred. Perhaps it is a result of some unknown link between perception and action (e.g.,
Kirsch \& Kunde, 2013) or, in this case, perception and spatial processing on one side and premotor planning and action on the other. Or, it may be due to the writing task the navigators performed at each destination: by passing the table on the left, they prevent their moving body from getting in the way of their right hand. In other words, going around the left side of the table is the shortest distance for the right hand. Future studies could focus on the conditions under which participants use a "left-side" heuristic, but our current focus is on the leastangle bias.

\section{General discussion}

On every trial in this study, participants walked to the same initial destination, choosing between the same two routes. The two routes themselves were very similar: they had the same length and the same initial angle with respect to the destination. As a result, there should have been a 50-50 chance that a particular route would be chosen. However, across three experiments the route participants selected varied in a consistent linear way with the locations of destinations they would later visit. This (new) "later-destination attractor" route choice bias is the tendency to take a route to the first destination that begins in the direction of subsequent destinations even when route choice to the first destination is logically independent of route choice to the other destinations.

The preferred routes to the first destination correspond generally to the least angle formed by the initial direction of the route and the direction of later destinations. The results of previous single-destination studies also showed that navigators prefer those routes that begin in the direction of their goal (Golledge, 1995). This least-angle bias has been demonstrated for route choices in small-scale environments (e.g., Bailenson et al., 1998, 2000) and in large-scale environments (e.g., Dalton, 2003). The current results are important because they confirm that there may be a common mechanism for route preferences regardless of the size of the space (e.g., Hicheur, 
Pham, Arechavaleta, Laumond, \& Berthoz, 2007; Waller \& Nadel, 2013); in this case, the common mechanism is the least-angle bias.

At the same time, the current experiments highlight the effects of memory load on route preferences. Specifically, as cognitive load increased, nearer destinations became the focus for the least-angle bias, and the overall least-angle bias decreased. Furthermore, any differences in route choice between small- and large-scale environments may be due more to cognitive load than to environmental size. It is likely that cognitive load would influence other aspects of spatial cognition, such as integrating different segments of a route (i.e., path integration; Allen et al., 2004; Meilinger, Berthoz, \& Wiener, 2011), and wayfinding in urban environments (Brunyé, Gardony, Mahoney, \& Taylor, 2012; Spiers \& Maguire, 2008) and multistory building environments (Raubal \& Egenhofer, 1998). Because many real-life navigation tasks involve varying degrees of cognitive load, it may be important to include cognitive load in future navigation research.

Perhaps the most important implication of this research relates to types of questions asked in navigation research. Previous work has tended to focus either on the sequencing of multiple destinations or to route choice to a single destination. Our experiments examined route choice to a single destination in an overall multiple-destination task. We found a robust effect of the direction of subsequent destinations on route choice towards the first destination. Given that the route navigators take to a destination can be strongly affected by where they plan to go next, this research suggests that more attention should be paid to route planning with multiple discrete yet sequenced destinations.

Acknowledgments We would like to thank Robrecht van der Wel and Bill Whitlow for their suggestions at the different stages of the study.

\section{References}

Allen, G. L., Kirasic, K. C., Rashotte, M. A., \& Haun, D. B. M. (2004). Aging and path integration skill: Kinesthetic and vestibular contributions to wayfinding. Perception \& Psychophysics, 66, 170-179.

Baddeley, A. (1992). Working memory. Science, 255(5044), 556-559.

Bailenson, N. J., Shum, S. M., \& Uttal, H. D. (1998). Road climbing: Principles governing asymmetric route choices on maps. Journal of Environmental Psychology, 18, 251-264.

Bailenson, N. J., Shum, S. M., \& Uttal, H. D. (2000). The initial segment strategy: A heuristic for route selection. Memory \& Cognition, 28(2), 306-318.

Brown, J. (1958). Some tests of the decay theory of immediate memory. Quarterly Journal of Experimental Psychology, 10, 12-21.

Brunyé, T. T., Gardony, A., Mahoney, R. C., \& Taylor, A. H. (2012). Going to town: Visualized perspectives and navigation through virtual environment. Computers in Human Behavior, $28(1), 257-266$.
Brunyé, T. T., Mahoney, C. R., Gardony, A. L., \& Taylor. (2010). North is up(hill): Route planning heuristics in real-world environments. Memory \& Cognition, 38(6), 700-712.

Christenfeld, N. (1995). Choices from identical options. Psychological Science, 6(1), 700-712.

Dalton, R. (2003). The secret is to follow your nose. Environment and Behavior, 35, 107-131.

Downs, R. M., \& Stea, D. (1973). Image and eenvironment. Chicago, IL: Aldine.

Evans, G. W., \& Pezdek, K. (1980). Cognitive mapping: Knowledge of real-world distance and location information. Journal of Experimental Psychology: Human Learning and Memory, 6, $13-24$.

Faul, F., Erdfelder, E., Lang, A.-G., \& Buchner, A. (2007). G*Power 3: A flexible statistical power analysis program for the social, behavioral, and biomedical sciences. Behavior Research Methods, 39, 175-191.

Gärling, T., Book, A., \& Lindberg, E. (1986). Spatial orientation and wayfinding in the designed environment: A conceptual analysis and some suggestions for postoccupancy evaluation. Journal of Architectural and Planning Research, 3(1), 55-64.

Golledge, R. G. (1995, May). Defining the criteria used in path selection. Paper presented at the conference Activity Based Approaches: Activity Scheduling and the Analysis of Activity Patterns, Eindgoven, The Netherlands.

Graham, S. M., Joshi, A., \& Pizlo, Z. (2000). The traveling salesman problem: A hierarchical model. Memory \& Cognition, 28(7), 1191-1204.

Hicheur, H., Pham, Q. C., Arechavaleta, G., Laumond, J. P., \& Berthoz, A. (2007). The formation of trajectories during goal-oriented locomotion in humans: I. A stereotyped behavior. European Journal of Neuroscience, 26(8), 2376-2390.

Hochmair, H. (2005). Investigating the effectiveness of the least-angle strategy for wayfinding in unknown street networks. Environment and Planning B: Planning and Design, 32, 673-691.

Hochmair, H. H., Büchner, S. J., \& Hölscher, C. (2008). Impact of regionalization and detour on ad-hoc path choice. Spatial Cognition \& Computation, 8(3), 167-192.

Hochmair, H., \& Frank, A. U. (2000). Influence of estimation errors on wayfinding-decisions in unknown street networks Analyzing the least-angle strategy. Spatial Cognition and Computation, 2, 283-313.

Hochmair, H., \& Karlsson, V. (2005). Investigation of preference between the least-angle strategy and the initial segment strategy for route selection in unknown environments. In C. Freksa, M. Knauff, B. Krieg-Bruckner, B. Nebel, \& T. Barkowsky (Eds.), Spatial cognition IV (LNAI 3343, pp. 79-97). Berlin, Germany: Springer.

Hölscher, C., Meilinger, T., Vrachliotis, G., Brösamle, M., \& Knauff, M. (2006). Up the down staircase: Wayfinding strategies in multi-level buildings. Journal of Environmental Psychology, 26(4), 284-299.

Kirsch, W., \& Kunde, W. (2013). Visual near space is scaled to parameters of current action plans. Journal of Experimental Psychology: Human Perception and Performance, 39(5), 1313-1325.

Lockman, J. J. (1984). The development of detour ability during infancy. Child Development, 55(2), 482-491.

MacGregor, J. N., Chronicle, E. P., \& Ormerod, T. C. (2004). Convex hull or crossing avoidance? Solution heuristics in the traveling salesperson problem. Memory \& Cognition, 32(2), 260-270.

MacGregor, J. N., \& Ormerod, T. (1996). Human performance on the traveling salesman problem. Perception \& Psychophysics, 58(4), 527-539.

Meilinger, T., Berthoz, A., \& Wiener, J. M. (2011). The integration of spatial information across different viewpoints. Memory \& Cognition, 39(6), 1042-1054. 
Montello, D. R. (1993). Scale and multiple psychologies of space. In A. U. Frank \& I. Campari (Eds.), Spatial information theory: A theoretical basis for GIS (pp. 312-321). Berlin, Germany: SpringerVerlag.

Peterson, L. R., \& Peterson, M. J. (1959). Short-term retention of individual verbal items. Journal of Experimental Psychology, 58, 193-198.

Presson, C. C., DeLange, N., \& Hazelrigg, M. D. (1989). Orientation specificity in spatial memory: What makes a path different from a map of the path? Journal of Experimental Psychology: Learning, Memory, and Cognition, 15, 887-897.

Raubal, M., \& Egenhofer, M. (1998). Comparing the complexity of wayfinding tasks in built environments. Environment \& Planning, 25(6), 895-913.

Roskos-Ewoldsen, B., McNamara, T. P., Shelton, A. L., \& Carr, W. S. (1998). Mental representations of large and small spatial layouts are viewpoint dependent. Journal of Experimental Psychology: Learning, Memory, and Cognition, 24, 215-226.

Scharine, A. A., \& McBeath, M. K. (2002). Right-handers and Americans favor turning to the right. Human Factors, 44, 248-256.

Shipstead, Z., Lindsey, D. R. B., Marshall, R. L., \& Engle, R. W. (2014). The mechanisms of working memory capacity: Primary memory, secondary memory, and attention control. Journal of Memory and Language, 72, 115-141.

Spiers, H. J., \& Maguire, E. A. (2008). The dynamic nature of cognition during wayfinding. Journal of Environmental Psychology, 28, 232249.
Timmermans, H., van der Hagen, X., \& Borgers, A. (1992). Transportation systems, retail environments and pedestrian trip chaining behaviour: Modelling issues and applications. Transportation Research Part B: Methodological, 26(1), 4559.

Vickers, D., Bovet, P., Lee, M. D., \& Hughes, P. (2003). The perception of minimal structures: Performance on open and closed versions of visually presented Euclidean travelling salesperson problems. Perception, 32, 871-886.

Vlamings, P. H. J. M., Hare, B., \& Call, J. (2010). Reaching around barriers: The performance of the great apes and 3-5-year-old children. Animal Cognition, 13, 273-285.

Waller, D., \& Nadel, L. (2013). Introduction: Frameworks for understanding spatial thought (or wrapping our heads around space. In D. Waller \& L. Nadel (Eds.), Handbook of spatial cognition (pp. 311). Washington, DC: APA Books.

Wiener, J. M., Ehbauer, N., \& Mallot, A. H. (2009). Planning paths to multiple targets: Involvement and planning heuristics in spatial problem solving. Psychological Research, 73(5), 644-658.

Wiener, J. M., \& Mallot, H. A. (2003). 'Fine-to-coarse' route planning and navigation in regionalized environments. Spatial Cognition and Computation, 3(4), 331-358.

Wiener, J. M., Schnee, A., \& Mallot, H. A. (2004). Use and interaction of navigation strategies in regionalized environments. Journal of Environmental Psychology, 24(4), 475-493. 